BABELAO 3 (2014), p. 125-144

C ABELAO (Belgium)

\title{
La linguistique marriste et son onomastique. Le cas de la Géorgie*
}

\section{Emmanuel Van Elverdinghe}

\author{
Aspirant F.R.S.-FNRS \\ Université catholique de Louvain, Louvain-la-Neuve
}

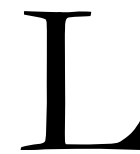

e développement des études caucasiennes en Russie impériale, puis en Union Soviétique, doit beaucoup à Nikolaj Jakovlevič Marr (1864-1934), savant prolifique et très créatif né de mère géorgienne, dont les théories en linguistique ont beaucoup évolué durant la carrière. ${ }^{1}$ Le terme « marrisme » a été forgé pour parler de l'ensemble des vues déve-

* Le noyau de cet article provient d'un mémoire de fin d'études, présenté à l'Université catholique de Louvain en juin 2013 et réalisé sous la direction du Pr Bernard Coulie. Nous le remercions vivement de son soutien à la rédaction du présent texte, qui a pu bénéficier de ses relectures et de ses conseils. Il va de soi que toute imperfection demeure pleinement de notre propre fait.

N.B. La translittération du géorgien dans cet article suit le système de la défunte Revue des études géorgiennes et caucasiennes, anciennement Bedi Kartlisa. Les auteurs et titres des ouvrages et articles cités ont cependant été maintenus dans leur orthographe d'origine.

${ }^{1}$ De nombreux travaux de N. Ja. Marr sont, aujourd'hui encore, d'un accès malaisé. Nous n'avons ainsi pas pu bénéficier de la lecture, entre autres, de son fascicule Кавказские племенные названия и местные параллели (Труды комиссии 
loppées par Marr et ses disciples. Marr lui-même prétendait avoir fondé une approche scientifique nouvelle, la «japhétidologie». Son ambition d'étendre cette approche, originellement élaborée pour la linguistique, à d'autres secteurs des sciences humaines, principalement l'ethnologie et l'archéologie, rencontra un succès limité. ${ }^{2}$ En linguistique en revanche, la domination de la japhétidologie au temps de Staline était pour ainsi dire totalitaire, son apport s'étendant depuis le développement d'alphabets pour les langues non écrites de l'Union jusqu'à la philosophie du langage et à la théorisation de la glottogenèse.

Cet article se structure en deux parties, l'une générale, l'autre spécifique. La première propose une esquisse de ce que fut la linguistique marriste, limitée à ses grandes lignes. Nous y distinguons dans un premier temps différentes étapes de la progression du marrisme ${ }^{3}$, ensuite plusieurs axes qui ont traversé toute son évolution. La seconde partie est consacrée à l'étude de l'onomastique par l'école de Marr. La place que ce dernier lui a accordée dans ses

по изучению племенного состава населения России 5), Pétrograd, 1922 [N. Ja. MARR, Les appellations des tribus du Caucase et leurs parallèles locaux (Travaux de la commission pour l'étude de la composition tribale de la population de Russie 5)]. En langue originale, il faut signaler ses CEuvres choisies (Н.Я. MAPP, Избранныце работыг, Léningrad / Moscou, 1933-1937, 5 vol.), qui ne reprennent malheureusement qu'une partie du considérable volume de ses publications. Une bibliographie complète en est présentée aux p. XI-XXVI du vol. 1 et XXI du vol. 5.

De bonnes présentations d'ensemble du marrisme sont fournies par les ouvrages de В. М. ААПАТОВ, История одного мифа: Марр и марризм [V. М. АLРАTOV, Histoire d'un mythe : Marr et le marrisme], Moscou, 1991 et R. L'HERMITTE, Science et perversion idéologique. Marr, marrisme, marristes. Une page d'histoire de la linguistique soviétique (I.M.E.S.C.O., Cultures et Sociétés de l'Est 8), Paris, 1987. Pour ce qui est du côté purement linguistique du marrisme, la référence reste L. L. Thomas, The Linguistic Theories of N. Ja. Marr (University of California Publications in Linguistics 14), Berkeley / Los Angeles, 1957. Enfin, il faut signaler qu'à l'initiative de l'Institut de Linguistique et des Sciences du Langage de l'Université de Lausanne, un colloque consacré à Marr s'est tenu du $1^{\text {er }}$ au 3 juillet 2004 à Crêt-Bérard (Vaud), dont les actes ont été rassemblés dans P. SÉRIOT (ed.), Un paradigme perdu : la linguistique marriste, Cabiers de l'ILSL 20 (2005); on y trouvera un excellent panorama de l'état actuel des recherches sur Marr, sous ses multiples facettes.

${ }^{2}$ Cf. Fr. Bertrand, «N. Marr et le marrisme pour l'ethnographie soviétique des années 1920-1930», Cabiers de l'ILSL 20 (2005), p. 27-38.

${ }^{3}$ Il s'agit d'une présentation chronologique volontairement simplifiée; nous renvoyons, pour un exposé complet du marrisme en linguistique, à THOMAS, Linguistic Theories, op. cit. L'on trouvera également davantage de détails dans l'ouvrage de M. CHERCHI - H. P. MANNING, Disciplines and Nations: Niko Marr vs. His Georgian Students on Tbilisi State University and the Japhetidology/Caucasology Schism (The Carl Beck Papers in Russian \& East European Studies 1603), Pittsburgh, 2002, ainsi qu'un aperçu similaire dans l'article de K. TUITE, «The Rise and Fall and Revival of the Ibero-Caucasian Hypothesis ", Historiographia Linguistica 35, 1/2 (2008), p. 23-82. Ces contributions nous ont servi de référence dans ce point, notamment pour la délimitation en périodes de l'activité scientifique de Marr. 
théories fait l'objet d'une brève présentation. La discussion porte ensuite sur les ethnonymes et toponymes de Géorgie, qui sont envisagés non directement selon les textes de Marr, mais bien au travers de reprises ultérieures des théories marristes. Cela permet également de montrer par divers exemples comment celles-ci sont réalisées dans la pratique. Nous espérons ainsi donner au lecteur à la fois un aperçu synthétique et illustré du marrisme et une idée de sa permanence dans les recherches récentes en études géorgiennes et caucasiennes.

\section{Regard général sur la linguistique marriste}

\section{Le parcours de N. Ja. Marr et du marrisme}

Aussi longtemps que les recherches en linguistique caucasienne sont restées principalement concentrées dans les métropoles européennes et russes, elles sont demeurées indifférentes aux problématiques locales, détachées en quelque sorte de leur terre nourricière, et dominées par l'optique néogrammairienne. L'indigénisation, légitime et nécessaire, de cette science, c'est-à-dire sa réappropriation par des personnes directement impliquées dans leur identité par ses résultats, a naturellement accéléré son essor, en même temps qu'elle l'a rendue susceptible d'interprétations plus originales et engagées. Concrètement, ce processus prend de l'ampleur à la fin du XIX ${ }^{\mathrm{e}}$ siècle à Saint-Pétersbourg puis, après la Révolution d'Octobre, se délocalise progressivement vers la Géorgie.

1884-1916. Dès son arrivée à l'université de Saint-Pétersbourg, Marr se distingua en décidant d'étudier simultanément toutes les langues proposées dans le cursus en orientalisme. Par la suite, il se spécialisa dans l'arménien et les langues kartvéliennes, surtout le géorgien et le laze. Très vite, il tenta d'apparenter ces dernières aux langues sémitiques en dégageant de leur lexique des racines triconsonantiques, ensuite reliées à de prétendus cognats sémitiques. Sous le vocable "japhétique», les langues kartvéliennes, accompagnées de certaines strates décelées dans l'arménien, se virent au terme de ces recherches définies comme une famille indépendante, mais proche de la famille sémitique.

1916-1920. Dans les autres langues du Caucase, Marr prétendit ensuite déceler des strates japhétiques, au nom de sa théorie de leur «mixité » historique. A partir de là, le terme "japhétique» s'étendit à l'ensemble des langues du Caucase, kartvéliennes ou non : abkhaze, bats (c'ova-tuš), tchétchène, avar etc. ${ }^{4}$

1920-1923. L'étape suivante fut d'élargir encore le champ en y incluant toutes ces langues qui, autour du bassin méditerranéen,

\footnotetext{
${ }^{4}$ Cf. Thomas, Linguistic Theories, op. cit., p. 39.
} 
ont toujours suscité la perplexité des linguistes : basque, étrusque, "pélasge ", langues asianiques et d'autres encore rentrèrent dans la famille japhétique. Déjà durant cette "phase méditerranéenne », Marr s'intéressait également à des langues plus éloignées, telles que l'élamite, le sumérien ou le bourouchaski (veršik). ${ }^{5}$

1923-1934. Durant la dernière période de l'activité de Marr, la japhétidologie s'appropria des langues du monde entier: breton, tchouvache, komi, hottentot, chinois, langues dravidiennes et bien d'autres furent étudiées dans leurs caractéristiques japhétiques, voire simplement annexées sans discussion à la japhétidologie. Le point culminant de cette évolution perpétuelle des idées de Marr, résolvant l'aporie née de l'extension sans fin de leur champ d'exercice, fut la «nouvelle théorie du langage » (новое учение о[б] языъке), qui proposait une vision marxiste de la linguistique, en rupture radicale avec le mode de pensée «bourgeois» de la linguistique indo-européenne, historique et comparatiste. Il n'était plus question d'une famille japhétique, mais d'un "système » japhétique, qui pouvait caractériser toute langue du monde à un stade donné de son évolution, cette dernière étant fonction de la lutte des classes. En d'autres termes, les liens fondamentaux unissant ces langues ne relevaient plus de la génétique, mais de la stratification sociale. Finalement, l'« analyse paléontologique » menée par Marr aboutit à des conclusions originales sur la genèse du langage parlé : tout serait parti de quatre " éléments primaires » à vocation magique, SAL, BER, YON et ROŠ. ${ }^{6}$

1934-1950. Erigée en dogme par le pouvoir soviétique des années 1920-1930, la nouvelle théorie du langage constitua dès lors le cadre impératif de toute recherche en linguistique en Union Soviétique. Il faut cependant reconnaitre que la personnalité de Marr était le véritable pilier du succès de sa doctrine : peu d'avancées furent faites par ses disciples - qui étaient d'ailleurs en

${ }^{5}$ A noter que l'ourartéen, langue des inscriptions cunéiformes du lac de Van, avait été étudié par Marr au titre de langue du Caucase et faisait donc partie des langues japhétiques de longue date (ibid., p. 39-40).

${ }^{6} \mathrm{Il}$ semblerait que nous puissions comprendre ces éléments comme des représentations conventionnelles de borborygmes primitivement indistincts, déduites à partir des noms de tribus "Sarmates ", "Ibères ", «Ioniens » et «Etrusques ». Toutefois, en réalité, Marr n'a jamais donné d'explication univoque et cohérente à ce choix, comme du reste à la plupart de ses assertions. Cf. notamment T. NiKOLAEVA, «Les "éléments primaires" chez les marristes et la complémentarité du paradigme linguistique », trad. du russe par E. Velmezova, Cabiers de l'ILSL 20 (2005), p. 215-226 : p. 216-219 et A. DuličENKO, «N. Marr à la recherche du sens du langage », trad. du russe par E. Velmezova, Cabiers de l'ILSL 20 (2005), p. 89-108: p. 94-95 et 100. Quelques exemples seront présentés au fil de cet article. 
nombre assez restreint - dans les années qui suivirent sa mort. ${ }^{7}$ On citera parmi ses principaux continuateurs son disciple Ivan Ivanovič Meščaninov (1883-1967). La nouvelle théorie fut officiellement récusée en 1950 par Staline, vraisemblablement sur les instances du linguiste géorgien Arnold Čikobava ${ }^{8}$, à la faveur d'une « discussion » dans la Pravda'. Elle suivit en somme la même trajectoire, avec une avance de quelques années, que la biologie marxiste de Lysenko; ces deux dérives sont souvent prises comme archétypes de la façon dont une idéologie totalitaire peut cadenasser la recherche scientifique dans le sens qu'elle entend.

Depuis 1950. L'intervention de Staline mit un coup d'arrêt définitif au développement du marrisme. La linguistique soviétique se trouva ainsi débarrassée d'un carcan encombrant. Cependant, rien n'aurait pu effacer subitement la trentaine d'années d'influence de Marr sur les esprits, ni ses écrits, quoique leur propagation fût sérieusement bridée. Si la nouvelle théorie du langage, dans son état pur, est restée largement confinée à l'Union Soviétique de Staline, l'on constate cependant une résurgence sporadique des idées ou des découvertes de Marr. Cela peut concerner à la fois les milieux scientifiques - nous y reviendrons dans la seconde partie de cet article - et certains cercles profanes. ${ }^{10}$

\section{Axes de développement du marrisme}

L'on distingue au travers de ce bref aperçu chronologique la constante évolution qui caractérise les idées de Marr. Il a été souligné par plusieurs que ses idées les plus tardives se trouvaient déjà en germe dès le début de son activité linguistique ; la logique de travail de Marr devait inexorablement mener à leur éclosion. ${ }^{11}$

\footnotetext{
7 Sur cette période, voir notamment Yu. SLEZKINE, « N. Ia. Marr and the National Origins of Soviet Ethnogenetics », Slavic Review 55, 4 (hiver 1996), p. 826-862 : p. 851-857.

${ }^{8}$ TUITE, Ibero-Caucasian Hypothesis, op. cit., p. 44 ; voir aussi SLEZKINE, Soviet Ethnogenetics, op. cit., p. 857-859.

${ }^{9}$ Edition du 20 juin 1950. Le texte de l'intervention de Staline a été plusieurs fois reproduit, augmenté et traduit. En russe, on lira par exemple И. СТАһИН, Марксизм и вопросы язвюкознания, s.l. (Moscou), 1953 ; en français, J. STALINE, Le marxisme et les problèmes de linguistique, Pékin, 1974.

10 Sur cette question, of. Vl. AlPATOV, «Que peut apporter l'héritage de Marr ? », trad. du russe par P. Sériot, Cabiers de l'ILSL 20 (2005), p. 11-26; M. SLODZIAN, "Actualité de Marr, ou permanence de l'utopie », Cabiers de l'ILSL 20 (2005), p. 271-293 ; CHERCHI - MANNING, Disciplines and Nations, op. cit., p. 54-55 et p. 64 n. 16.

11 Thomas, Linguistic Theories, op. cit., p. 61, 135-136 e.a.; W. K. MATTHEWS, "The Japhetic Theory », The Slavonic (and East European) Review 68 (décembre 1948, vol. 27), p. 172-192 : p. 176-177 ; SLEZKINE, Soviet Ethnogenetics, op. cit., p. 834 e.a.
} 
C'est ainsi que Marr s'est montré toujours plus catégorique et extrémiste dans son rejet de la méthode comparative traditionnelle telle que pratiquée par les indo-européanistes, qu'il considérait méprisants envers les petites langues - dont sa langue maternelle, le géorgien. Cela dit, il ne s'en inspirait pas moins, dans une dynamique d'attraction-répulsion. ${ }^{12}$ Le plus flagrant à cet égard est que tout en s'en distanciant explicitement, Marr empruntait à la grammaire comparée indo-européenne ses outils et sa terminologie - en apparence seulement, comme nous le verrons ci-dessous. De même, la diachronie élaborée par Marr à toutes les étapes de sa carrière s'avère en réalité fondamentalement anhistorique. ${ }^{13}$ Allant totalement à l'encontre du structuralisme saussuréen, il n'établissait aucune distinction entre synchronie et diachronie - quoique lui-même prétendît exactement le contraire ${ }^{14}-$, en considérant que les langues sont toutes issues d'une longue histoire de croisements complexes les unes avec les autres, malgré lesquels tous les éléments linguistiques primordiaux, immortels, ainsi que leur histoire mouvementée, se laissent encore distinguer en tant que tels dans chaque mot à chaque époque.

De ce fait, un aspect évident du marrisme est pour nous son manque de scientificité. La japhétidologie mettait en jeu, aux dires de Marr, un arsenal de «lois» phonétiques, de "correspondances » et d'«alternances » « régulières ». Il s'agit là d'une terminologie extrêmement récurrente dans ses travaux; seulement, ces principes méthodologiques n'ont jamais été publiés. ${ }^{15}$ Cet état de fait ne concerne pas seulement sa linguistique formelle, mais aussi sa sémantique. ${ }^{16}$ Bien plus, le lecteur de Marr doit faire face à un nombre incalculable d'incohérences, de contradictions, de volteface et d'erreurs manifestes dans la mise en œuvre de cette mé-

12 Expression reprise à SLODZIAN, Actualité de Marr, op. cit., p. 277-278 ; voir aussi CHERCHI - MANNING, Disciplines and Nations, op. cit., p. 6-8 et 14-17 et AlPATOV, L'Héritage de Marr, op. cit., p. 16.

13 ThOMAS, Linguistic Theories, op. cit., p. 49-52 (voir aussi p. 28-29, 42 e.a.)

14 Ibid., p. 52 et O. LEŠČAK - Ju. SITKO, "Les considérations ontognoséologiques de Marr du point de vue de la méthodologie pragmaticofonctionnelle », trad. du russe par E. Simonato et P. Sériot, Cabiers de l'ILSL 20 (2005), p. 177-198 : p. 192.

${ }^{15}$ CHerchi - MANNING, Disciplines and Nations, op. cit., p. 21. Même là où l'on s'y attendrait, ces principes ne sont pas exprimés clairement: voir H.Я. MAPр, Обший курс учения об языке [N. Ja. MARR, Cours général de théorie du langage], repris dans ID., Избранныз работье, op. cit., vol. 2, p. 12-126: \10= p. 37-38 (traduction allemande en p. 107-110 de N. Ja. MARR, Die japhetitische Theorie, trad. du russe par T. Borbé, in T. BORBÉ, Kritik der marxistischen Sprachtheorie N.Ja. Marr's (Skripten Linguistik und Kommunikationswissenschaft 8), Kronberg im Taunus, 1974, p. 63-262).

16 Voir à ce sujet E. VeLMEZOVA, Les lois du sens: la sémantique marriste (Slavica Helvetica 77), Berne, 2007, p. 294-296 e.a., ainsi que THOMAS, Linguistic Theories, op. cit., p. 68 sqq. 
thode. ${ }^{17} \mathrm{Il}$ a enfin été montré que même dans ses travaux généralement considérés comme sérieux, comme sa grammaire du géorgien ancien ${ }^{18}$, Marr avait tendance à arranger les faits selon ce qui lui convenait. ${ }^{19}$

Cela dit, s'agissant des théories de Marr, il faut distinguer validité scientifique et validité philosophique. Mal connues en dehors de l'Union Soviétique, ses idées ont été valorisées à l'intérieur de celle-ci sur la seule base de leur conformité perçue à l'idéologie nationale. C'est d'ailleurs sur ce point que portera la critique de Staline, non sur de véritables "problèmes de linguistique ». Crédit et discrédit du marrisme furent donc fonction du programme idéologique du pouvoir soviétique, qui se souciait bien peu de sa fiabilité scientifique.

Un aspect capital du mode opératoire de Marr doit encore être mis en évidence. Il est en effet patent que ses recherches étaient orientées a priori : c'est en fonction de ce qu'il cherchait à prouver que Marr se mettait en quête de preuves ad hoc, ne sélectionnant que les faits ou les interprétations qui allaient dans le sens de ses idées préétablies. ${ }^{20} \mathrm{Et}$, a minori, ses théories elles-mêmes répondaient à ses exigences du moment, ce qui explique leurs multiples incohérences et contradictions. ${ }^{21}$ Naturellement, un tel mode de fonctionnement convenait à merveille à son intuition remarquable.

Le potentiel de Marr était effectivement indiscutable ; ses professeurs à Pétersbourg et ceux qui l'ont fréquenté du temps de son activité ne s'y sont pas trompés ${ }^{22}$, et nombreux, même parmi ses détracteurs, sont ceux qui lui ont reconnu de géniales intuitions. Ce qui l'a perdu, c'est de n'avoir su s'imposer aucune bride, et le fait qu'il n'ait jamais reçu de formation spécifique en linguistique n’y est probablement pas étranger. ${ }^{23}$ Ses théories étaient

${ }^{17}$ Ibid., p. 135 et illustrations passim (p. ex. p. 66-68).

18 N. MARR - M. BriÈre, La langue géorgienne, Paris, 1931.

${ }^{19}$ AlPATOV, L'Héritage de Marr, op. cit., p. 17-18 ; comme l'auteur le laisse entendre (peut-être malgré lui), cela ne peut manquer de jeter un certain discrédit sur sa grammaire laze (Н. МАРРъ, Грамматика чанскаго (лазскаго) языка. Съ хрестоматіего и словаремъ (Матеріалы по яфетическому языкознанію 2) [N. MARR", Grammaire de la langue tch'ane (laze). Avec une chrestomathie et un glossaire (Matériaux de linguistique japhétique 2)], Saint-Pétersbourg, 1910), qui est de nos jours son œuvre la plus estimée. Voir aussi THOMAs, Linguistic Theories, op. cit., p. 10-11.

20 AlPatov, L'Héritage de Marr, op. cit., p. 17.

21 Thomas, Linguistic Theories, op. cit., p. 135.

22 Voir notamment ibid., p. 2-3.

${ }^{23}$ Sa seule rencontre avec des linguistes professionnels (Hübschmann, Jensen etc.), lors d'un voyage d'études à Strasbourg, fut d'ailleurs désastreuse (ibid., p. 1-4). 
ainsi appuyées par des étymologies de plus en plus extravagantes, ce manque de rigueur desservant de manière irréparable sa créativité, qui n'était pas répréhensible per $s .^{24}$ En résumé, Marr faisait intervenir des démarches et concepts scientifiques parfaitement recevables, mais les pervertissait en les mettant au service d'idées préconçues, sans jamais se préoccuper d'établir une méthodologie critique. $^{25}$

\section{L'onomastique marriste et ses échos}

\section{L'onomastique dans les théories de N. Ja. Marr}

La question des ethnonymes et des toponymes revêt une importance capitale dans la pensée de Marr, étant donné que ses théories en linguistique générale et en paléontologie du langage découlent directement de l'étude de tels termes. ${ }^{26}$ Le poids excessif qu'il leur accordait a d'ailleurs été souligné de longue date. ${ }^{27} \mathrm{Se}$ baser sur des étymologies de noms propres pour établir des correspondances linguistiques est en effet extrêmement risqué d'un point de vue scientifique ${ }^{28}$; d'un autre côté, l'impénétrabilité étymologique des ethnonymes et des toponymes, comme le fait remarquer K. Tuite ${ }^{29}$, a pour contrecoup la facilité avec laquelle ils peuvent être soumis aux préjugés de qui les analyse. Mieux sans doute que tout autre domaine où s'est exercé son esprit, l'étymologie des ethnonymes et des toponymes révèle les fondements programmatiques, et non empiriques, de la méthode de Marr et de ses «découvertes». Celles-ci, de façon systématique, inté-

${ }^{24}$ Vues sous un angle symbolique, voire ésotérique, les étymologies de Marr ne sont ainsi pas dénuées d'intérêt, comme le dépeint R. TRIOMPHE, «La mythologie «japhétique»: Marr entre le Caucase, la Bible et la Grèce », Cabiers de l'ILSL 20 (2005), p. 311-341 : p. 340.

25 TUITE, Ibero-Caucasian Hypothesis, op. cit., p. 44.

${ }^{26}$ Cf. ThOmas, Linguistic Theories, op. cit., p. 62-66, 72 et passim.

${ }^{27}$ Déjà par Šavaxišvili en 1937 (CHERCHI - MANNING, Disciplines and Nations, op. cit., p. 21-22). Voir également, entre autres, L. L. THOMAS, «Some Notes on the Marr School », American Slavic and East European Review 16, 3 (octobre 1957), p. 323-348 : p. 327-330.

${ }^{28}$ Cf. p. ex. la note de V. PISANI, L'etimologia. Storia - questioni - metodo, 2a ed. riveduta e accresciuta, Brescia, 1967, p. 134 n. 16. Voir aussi И.М. АьяКОНОВ, Предвгстория армянского народа. История арманского нагорья с 1500 по 500г. до н.э. Хурриты, Аувийци, Протоармане [I. M. D’JAKONOV, La prébistoire du peиple arménien. Histoire du haut plateau arménien de 1500 à 500 av. n. è. Hourrites, Louvites, Proto-Arméniens], Erevan, 1968, p. 14 et n. 13, ainsi que p. 194-197 ; B. FALCKKJÄLLQUIST, «On proper names in historical-etymological dictionaries, with special reference to personal names ", in J.-P. CHAMBON (ed.) e.a., Onomastik und Lexikographie, Deonomastik (Akten des 18. Internationalen Kongresses für Namenforschung, Trier, 12. - 17. April 1993, Bd. 5 / Patronymica romanica 18), Tübingen, 2002, p. 33-40.

29 TUITE, Ibero-Caucasian Hypothesis, op. cit., p. 42-43 n. 26. 
ressent ses thèses - établies au préalable - et «confirment» ses présupposés.

Comme pour tous les éléments du langage, Marr a voulu réduire les ethnonymes et les toponymes à des racines, dont le nombre et la complexité sont allés décroissant : douze en 19191920, elles ne sont plus que quatre en 1926, les racines de plus de deux consonnes ou au phonétisme proche ayant progressivement été fondues dans celles qui restent. ${ }^{30}$ Ces quatre racines primitives SAL, BER, YON et ROŠ, que nous avons déjà mentionnées et sur lesquelles repose toute la paléontologie élaborée dans le cadre de la nouvelle théorie du langage, sont donc en dernière analyse dérivées d'éléments ethno-toponymiques, analysés par Marr depuis 1912-1913 sous la forme préfixe(s) + CVC + suffixe $(s)^{31}$ : p. ex.,

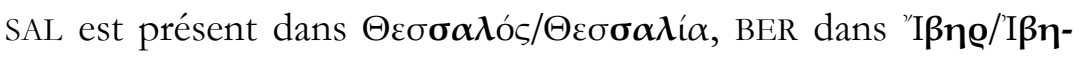

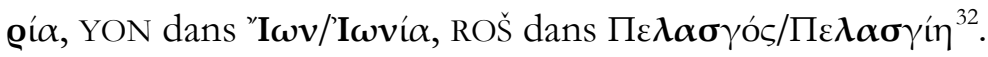

Cependant, il n'y a pas que la morphologie qui compte : Marr a également consacré beaucoup d'énergie à la mise au point d'une sémantique historique propre à sa théorie, où les ethniques tiennent une place particulière. ${ }^{33}$ Selon lui, l'homme primitif appréhendait le monde au moyen de concepts extrêmement englobants et indistincts. Au fur et à mesure du développement cognitif de l'humanité, ces concepts se sont affinés par dichotomies successives, l'homme distinguant, classant, différenciant ce qui l'entoure avec une précision toujours accrue. D'évidence, ce développement putatif du signifié marrien est parallèle à celui du signifiant : de la même façon que toutes les formes de toutes les langues sont ramenées à un nombre réduit de racines primitives, tous les sens remontent à quelques sens primitifs universels. ${ }^{34}$ Or, toujours selon Marr, dans la pensée magique qui caractérisait l'homme primitif, ces concepts étaient associés à des puissances divines, concrètement représentées par des totems. Chaque tribu, vénérant particulièrement un certain totem, aurait fini par endosser

30 ThOMAS, Linguistic Theories, op. cit., p. 62-63.

${ }^{31}$ Ibid., p. 44.

${ }^{32}$ Hdt. II, 56. Ces exemples et d'autres dans THOMAs, Linguistic Theories, op. cit., p. 62. Le cas d'I $\beta \eta \emptyset$ et 'I $\beta \eta{ }^{\prime} \alpha$ sera exploré plus en détail infra.

${ }^{33}$ Sur la sémantique marriste, on consultera en priorité VELMEZOVA, Les lois $d u$ sens, op. cit. Pour les développements qui suivent, $c f$. notamment THOMAS, Linguistic Theories, op. cit., p. 69 sqq., DulIČENKO, Recherche du sens, op. cit., p. 97100 et, en résumé, E. VeLmezovA, «Peuples et langues slaves : une "aberration" de la «linguistique traditionnelle »? La slavistique fantastique de N.Ja. Marr », Cahiers de l'ILSL 26 (2009), p. 187-198 : p. 191-192 n. 34.

${ }^{34}$ La sémantique de Marr a d'ailleurs évolué simultanément à sa linguistique formelle, se transformant en même temps que la nouvelle théorie du langage était proclamée (THOMAS, Linguistic Theories, op. cit., p. 72). Cependant, les bases et orientations théoriques de cette sémantique, auxquelles nous nous limitons, ont peu varié. 
elle-même le nom de ce totem, sous la forme en usage à l'intérieur de la tribu.

L'idée d'un tel processus "totémique ", qui était dans l'air du temps au début du $\mathrm{Xx}^{\mathrm{e}}$ siècle ${ }^{35}$, s'avère psychologiquement, anthropologiquement et typologiquement tout à fait recevable. La linguistique ne s'y oppose pas : l'on peut faire remonter un certain nombre d'ethnonymes et de toponymes à des noms d'animaux, catégorie totémique par excellence. ${ }^{36}$ Toutefois, il importe de remarquer, d'une part que la limite avec l'étymologie populaire est parfois ténue, d'autre part que, comme nous l'apprend l'ethnoanthropologie, le groupe distingué par un totem n'est pas nécessairement de nature ethnique, ni même tribale : il s'agit bien souvent d'un clan, d'une catégorie sociale, voire d'un groupement d'une autre nature encore, sans aucun lien avec la filiation. ${ }^{37}$

\section{Répercussions dans les travaux de Cytille Toumanoff}

Eminent spécialiste du Caucase chrétien, le prince Cyrille Toumanoff (1913-1997) fut sans conteste l'un des savants les plus respectés dans ce domaine, qu'il a largement contribué à faire connaitre aux milieux scientifiques occidentaux. ${ }^{38}$ L'histoire politique et dynastique de la Géorgie et de l'Arménie médiévales constituait son domaine de spécialité, ce qui l'a naturellement conduit à s'intéresser également à la préhistoire de ces régions,

35 Ainsi W. WundT, Elemente der Völkerpsychologie. Grundlinien einer psychologischen Entwicklungsgeschichte der Menschheit, zweite, unveränderte Auflage, Leipzig, 1913, p. 117-278 («Das totemistische Zeitalter») ou S. FREUD, Totem und Tabu. Einige Übereinstimmungen im Seelenleben der Wilden und der Neurotiker, Leipzig / Vienne, 1913, p. 92-149 («Die infantile Wiederkehr des Totemismus»), pour ne citer qu'eux, reconstituent-ils comme Marr un processus de nominalisation des tribus primitives par adoption d'un totem. Marr lui-même se revendiquait des travaux du sociologue français Lucien Lévy-Bruhl.

${ }^{36}$ Par exemple la tribu samnite des Hirpini, de birpus «loup » (A. WALDE J. B. Hofmann, Lateinisches etymologisches Wörterbuch, 4. Auflage, 1965-1972, 3 vol., s.v. hircus), ou le toponyme mésopotamien Lagaš, qui pourrait bien être le nom d'une espèce de corbeau, symbole de la ville (cf. Th. JaCOBSEN, "Some Sumerian City-Names », Journal of Cuneiform Studies 21 (1967), p. 100-103 et, en dernier lieu, G. WhITTAKER, «The Sumerian Question: Reviewing the Issues », in W.H. VAN SOLDT, R. KALVELAGEN et D. KATZ (edd.), Ethnicity in Ancient Mesopotamia. Papers Read at the 48th Rencontre Assyriologique Internationale, Leiden 1-4 July 2002 (PIHANS 101), Leyde, 2005, p. 409-429 : p. 416). Des totems inanimés sont plus rares, mais il s'en trouve, p. ex. " eau coulant du toit», "igname grimpant» ou «cœur» chez les Ganda d'Ouganda (voir le relevé de M. B. NsIMBI, Luganda Names, Clans and Totems (Munger Africana Library Notes 52/53), Pasadena, 1980).

${ }^{37}$ Cf. Cl. LÉvi-STRAuss, Le totémisme aujourd'bui, 2e éd., Paris, 1965, spécialement p. 64 sur les différents types de totémisme.

${ }^{38}$ Sur la vie et la carrière de Cyrille Toumanoff, voir N. G. GARSOÏAN, « In memoriam Cyrille Toumanoff », Revue des Etudes Arméniennes 26 (1996-1997), p. 457-458 et R. H. HEWSEN, "In Memoriam: Cyril Toumanoff », Journal of the Society for Armenian Studies 8 (1995), p. 5-7. 
sans toutefois qu'il y mène une recherche originale d'importance. Ses positions dans ce domaine nous intéressent particulièrement, en ce qu'elles reprennent certaines thèses marristes.

Une "note supplémentaire ", à la fin du premier volet de son introduction à l'histoire de la Caucasie chrétienne ${ }^{39}$, fait le point sur «The 'Third' Stratum of Mediterranean Civilization ». Ce titre réfère à un ouvrage de Marr, publié durant sa période méditerranéiste. ${ }^{40}$ La note de Toumanoff vise essentiellement à questionner l'importance de l'élément indo-européen dans la société hittite. Il y qualifie de «systems of great comprehensiveness and complexity that can be described as maximalist interpretations of the available data ${ }^{41}$ les théories élaborées par Marr et Joseph Karst au sujet du substrat non indo-européen autour de la Méditerranée, tout en précisant que «it is not necessary to subscribe to the Marxian linguistics of Marr's final phase [...] in order to admit as at least possible the theory elaborated by some of that school $[\ldots] »^{42}$. L'on peut dire que dans l'ensemble, comme le montre cette dernière citation, Toumanoff reste relativement prudent à l'égard du marrisme.

Dans un article daté de 1943 - c'est-à-dire avant la critique officielle du marrisme -, le savant se montrait, en expliquant à ses lecteurs le terme "Japhetite", plus catégorique sur la valeur des théories marristes. ${ }^{43}$ Par exemple, après avoir exprimé sa perplexité envers les développements marxistes ultérieurs de celles-ci, Toumanoff écrivait alors "That part of the theory, however, which admits of verification by history and philology and which is based on strict historical and philological research, is beyond all doubt of a very great value and significance. » Malheureusement, l'auteur n'indique pas précisément en quoi consiste, selon lui, « cette partie de la théorie » de Marr.

39 Publié sous forme d'article en 1959 : C. ToumANOFF, «Introduction to Christian Caucasian History. The Formative Centuries (IVth-VIIIth) », Traditio 15 (1959), p. 1-106 : p. 92-95.

${ }^{40}$ Н. Я. МАрР, Яфетический Кавказ и третий этнический элемент в созидании средиземноморской культуры (Материалы по яфетическому языкознанию 11), Leipzig, 1920 (repris dans ID., Избранные работы, ор. cit., vol. 1, p. 79-124). Cette brochure, légèrement révisée, a fait l'objet d'une traduction allemande : N. MARR, Der japhetitische Kaukasus und das dritte ethnische Element im Bildungsprozess der mittelländischen Kultur, übersetzt von F. Braun (Japhetitische Studien zur Sprache und Kultur Eurasiens 2), Berlin, 1923.

41 Toumanoff, Formative Centuries, op. cit., p. 92.

42 Ibid., p. 94.

${ }^{43}$ C. TOumanofF, « Medieval Georgian Historical Literature (VIIth-XVth centuries) », Traditio 1 (1943), p. 139-182 : p. 143 n. 8. Cet article vient d'être réimprimé dans S. H. RAPP, Jr. - P. CREGO (edd.), Languages and Cultures of Eastern Christianity: Georgian (The Worlds of Eastern Christianity, 300-1500 5), Farnham (Surrey) / Burlington (VT), 2012, p. 277-320. 
Dans la suite de cette note, Toumanoff évoque surtout les arguments linguistiques en faveur de ce substrat circumméditerranéen. Et nous touchons là au problème : historien de valeur, polyglotte chevronné, le prince Toumanoff n'était en revanche aucunement linguiste. Cela l'a conduit à accepter et à diffuser les étymologies marristes, sans leur appliquer d'autre critique que celle de son bon sens. ${ }^{44}$

Prenons le toponyme Ißßpé́ $\alpha$, courant dans les sources grecques pour désigner le Kartli. Toumanoff le rapporte à une racine \& $\mathrm{B}-\mathrm{L}$ or (through the mutation $l>r$ ) $\mathrm{B}-\mathrm{R}[\ldots]$ recognizable

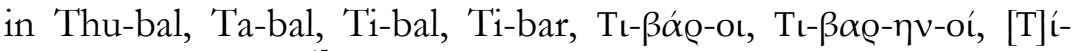
$\beta \eta \varrho-\varepsilon \varsigma$, II- $\beta \eta \varrho-i ́ \alpha »{ }^{45}$. D'emblée, dégageons les traits clairement marristes : la «mutation $l>r$ », fréquemment invoquée par Marr, l'absence totale de prise en considération des voyelles ainsi que les crochets autour du $[\mathrm{T}]$, qui permettent, sans la moindre expli-

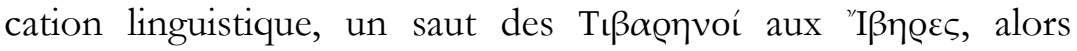

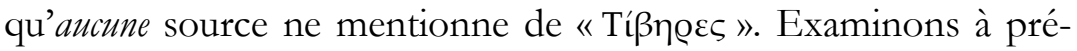
sent chacune des formes citées. Toumanoff enchaine d'abord le nom d'un personnage biblique (Tubal [תָרָ, quième fils de Japhet selon Gn 10, 2 et $1 \mathrm{Ch}$ 1, 5), le nom assyrien d'un pays louvite (Tabal, région occupée par plusieurs états néohittites et correspondant grosso modo à la Cappadoce), son équivalent hittite (Tibal) et une variante de celui-ci (Tibar). Il cite ensuite le nom, attesté par plusieurs auteurs classiques (Hdt., Xen. e.a.), d'un peuple du Pont, d'abord dans une graphie erronée (une par-

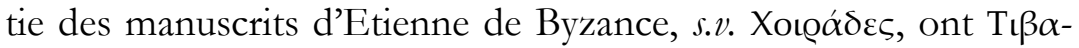

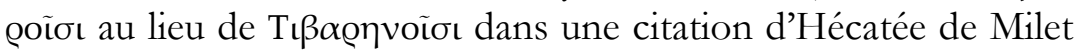

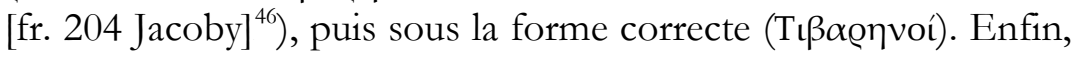

${ }^{44}$ Ce bon sens est par exemple exercé dans le cas du choronyme odgrngono imereti, dont Toumanoff refuse la dérivation à partir d'une base « G-R » ou « B$\mathrm{R} »(c f$. 'I $\beta \eta \mathrm{Q})$ : ibid., p. 146 n. 22 et C. TOUMANOFF, «The Social Background of Christian Caucasia », in ID., Studies in Christian Caucasian History, Washington (D.C.), 1963, p. 33-139 : p. 61 n. 58. Le nom odg ๓̆gono signifie en réalité «le pays (-gono -eti) au-delà (od[o]gங im[i]er) [de la chaîne de Lixi] ", par opposition au Junnmo kartli où cette désignation a vu le jour ( $c f$. G. DEETERS, «Der

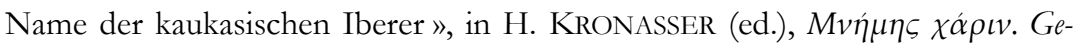
denkschrift Paul Kretschmer, Vienne, 1956, vol. 1, p. 85-88 : p. 85-86 et, plus récemment, S. H. RAPP, Jr., Studies in Medieval Georgian Historiography: Early Texts and Eurasian Contexts (CSCO 601 - Subsidia 113), Louvain, 2003, p. 439 e.a.). Marr jugeait cette étymologie impossible (Н. МАРРЬ, « Крещеніе армянъ, грузинъ, абхазовъ и алановъ святымъ Григоріемъ (Арабская версія) », Записки Восточнаго Отдъленія Императорскаго Русккаго Археологическаго Общества [N. MARR", "Le baptême des Arméniens, des Géorgiens, des Abkhazes et des Alains par saint Grégoire (version arabe) », Notes de la section orientaliste de la Société archéologique impériale de Russie] 16 (1904-1905), p. 63-211 : p. 167-168).

45 ToumanofF, Social Background, op. cit., p. 60 n. 58.

$46 \mathrm{Il}$ est à remarquer que la leçon correcte avait déjà été retenue en 1849 par Meineke dans son édition d'Etienne de Byzance. Marr ne pouvait donc manquer de connaitre le véritable statut de cette forme. Plus étonnant encore, Toumanoff la conserve lui aussi telle quelle (ibid., p. 57 n. 53). 


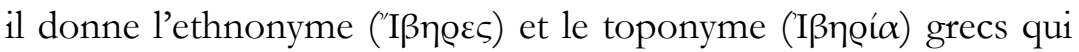
se rapportent au Kartli et parfois, lato sensu, à la Géorgie.

Le problème de cette chaîne d'équivalences réside dans la faiblesse croissante de ses maillons. La première équivalence posée, entre Tubal et Tabal/Tibal, est généralement acceptée : dans le livre d'Ezéchiel (27, 13 ; 32, 26 ; etc.), Tubal est le nom d'un pays, qui correspond au Tabal des sources assyriennes. ${ }^{47}$ Selon l'opinio

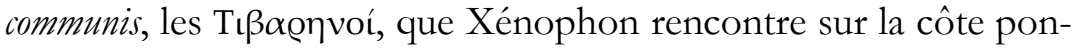
tique, sont les héritiers, refoulés vers le nord, des habitants de $\mathrm{Tabal}^{48}$, mais cette identification n'apparait pas totalement satisfaisante. ${ }^{49}$ L'équation de ces Tı $\beta \alpha$ @qvó́ avec les Ibères pose davantage problème. En effet, sur la foi de l'étymologie marriste ${ }^{50}$,

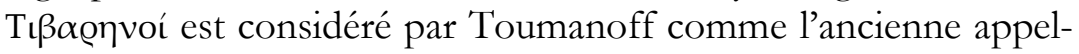
lation des 'I $\beta \eta \varrho \varepsilon s$, avant leur migration depuis le Pont vers le Kartli. ${ }^{51}$ Pourtant, aucun auteur ancien ne témoigne de cela : Strabon, qui connaît les deux populations, ne les apparente nullement. ${ }^{52}$ Certes, il existe chez Flavius Josèphe un passage (ant. I, 125 :

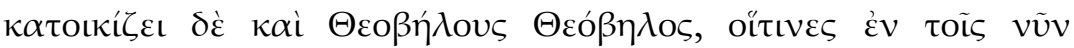

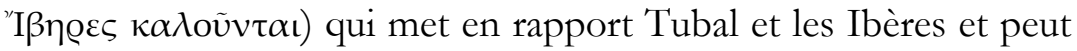
donc avoir inspiré cette série d'étymologies ; cependant, non seulement Josèphe ne dit pas de quels Ibères il parle, mais de plus, il

${ }^{47}$ H. Craig-Melchert (ed.), The Luwians (Handbuch der Orientalistik I, 68), Leyde / Boston, 2003, p. 97. Voir aussi Is 66, 19. La tradition juive n'a pas toujours situé Tubal au même endroit : ainsi, selon le livre des Jubilés (IX, 12), il occupe le sud-est de l'Europe, de la Roumanie au sud de la France (P. S. ALEXANDER, " Notes on the "Imago Mundi" of the Book of Jubilees », Journal of Jewish Studies 33 (1982), p. 197-213 : voir surtout p. 209 et la carte p. 213).

48 Outre Toumanoff, $c f$. p. ex. E. DHORME, «Les peuples issus de Japhet d'après le chapitre X de la Genèse », Syria, 13 (1932), p. 28-49 : p. 37-39 et, plus récemment, R. DREWS, "Herodotus 1.94, the Drought ca. 1200 BC, and the Origin of the Etruscans », Historia: Zeitschrift für alte Geschichte 41 (1992), p. 1439 : p. 18.

49 Voir D'JAKONOV, Предъзстория, ор. cit., p. 192-193 et 217-219.

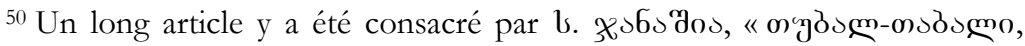

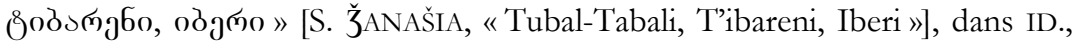

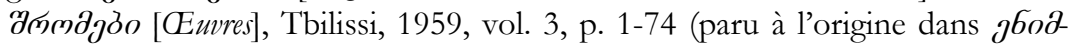

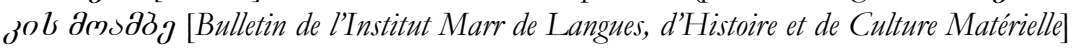
1 (1937), p. 185-245), accompagné d'un résumé en russe p. 75-80.

51 Toumanoff, Social Background, op. cit., p. 56-57 et n. 53. La datation de cette migration est très vague (« environ un siècle plus tard» que «les cinquième et sixième siècles »), et nulle part expliquée. La plus ancienne attestation certaine du grec 'I $\beta \eta \emptyset \varepsilon \varsigma$ pour désigner un peuple géorgien date du Ier siècle a.C.n., chez Strabon (voir notre article «Les Géorgiens dans les textes byzantins jusqu'à l'an mille. Approche lexicale », à paraître dans Byzantion 84 (2014)).

${ }^{52}$ Les Tibaréniens sont notamment mentionnés en geogr. XII, 3, 28 : v́tį̀

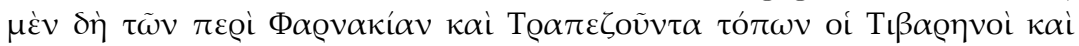

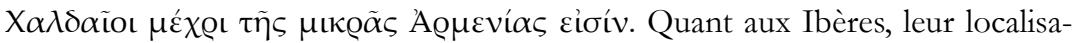
tion selon geogr. XI, 2, $19-3,6$ correspond au Kartli. Nulle part ils ne sont mentionnés conjointement. 
ne s'agit nullement d'un témoignage direct. Toumanoff voit dans

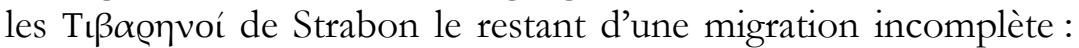
c'est possible, mais aucun argument, en dehors de cette hypothèse linguistique hasardeuse, ne permet de le prouver. ${ }^{53}$

Par ailleurs, Marr avait assigné le nom biblique «Tubal-Caïn» au groupe constitué par le mingrélien et le laze au sein de la famille kartvélienne et généralement appelé aujourd'hui "zan », dénomination elle-même peu heureuse ${ }^{54}$. Selon lui, Tubal et tous les noms que nous venons d'examiner étaient représentatifs de la préhistoire mingrélienne, et non du Kartli. Quant à Caïn, Marr y rattachait la racine z̧งb- "̌́an-, qui forme le nom géorgien des

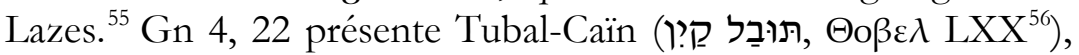
fils de Lamech - lui-même descendant de Caïn - et Tsilla, comme «l'ancêtre de tous les forgerons en cuivre et en fer ${ }^{57}$. Marr donne raison à la Bible en considérant ses «Tubal-Caïn » comme un peuple hybride, dont le premier élément a donné le nom du cuivre, et le second, du fer. ${ }^{58} \mathrm{Il}$ étaie par cet argument à la fois la parenté incontestable entre langues mingrélienne et laze et son idée que la métallurgie, avant de se répandre tout autour de la Méditerranée, serait originaire du Caucase japhétique. ${ }^{59}$

Pour Toumanoff, ce terme rend compte de la proximité qui devait jadis unir le groupe de peuples dont les noms sont issus de la racine B-L et ceux qui remontent à une racine $\check{C}-\mathrm{N} / \mathrm{S}-\mathrm{N} / \mathrm{H}-\mathrm{N} .{ }^{60}$

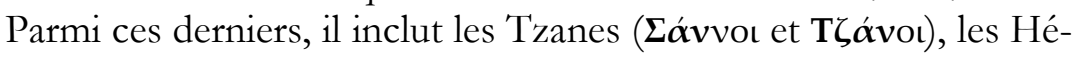
nioques (Hvíoxoı) et le deuxième élément présent chez les Mossy-

${ }^{53}$ Le récent travail de RAPP, Medieval Georgian Historiography, op. cit., admet le lien historique établi de la sorte entre Tubal et le Caucase ; l'auteur montre toutefois son absence dans l'historiographie médiévale géorgienne (voir p. 136-137 n. 139 et p. 139).

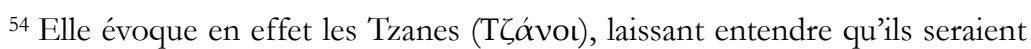
les ancêtres communs des Mingréliens et des Lazes, ce qui n'est absolument pas le cas.

55 Cf. Thomas, Linguistic Theories, op. cit., p. 45 et 151 n. 63.

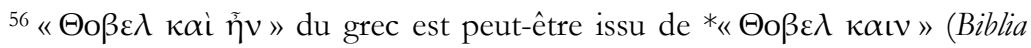
Hebraica, ed. R. KITTEL e.a., 10e éd., Stuttgart, 1937, p. 6 in app.).

57 Traduction de la Bible de Jérusalem.

58 MARR, Яфетический Кавказ, ор. сіt., in ID., Избранные работы, ор. cit., vol. 1, p. $104-105$ et 112 .

${ }^{59}$ Ibid., p. 118-120. Voir TrIOMPHE, Mythologie «japhétique», op. cit., p. 315 et 325 sqq. au sujet de l'inspiration biblique de Marr. Un peu plus tard, Marr considérera que l'apparition de la métallurgie, occasionnant des bouleversements dans la structure socio-économique des peuples concernés, avait fait basculer leurs langues du stade japhétique vers le stade prométhéen, i.e. indo-européen (THOMAS, The Marr School, op. cit., p. 330 et 333).

${ }^{60}$ Toumanoff, Formative Centuries, op. cit., p. 20 n. 27 ; ID., Social Background, op. cit., p. 58 n. 55 . Voir aussi ibid, p. 60-61 n. 58. 


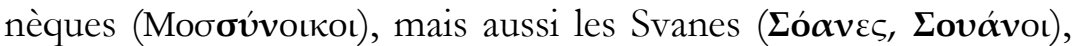

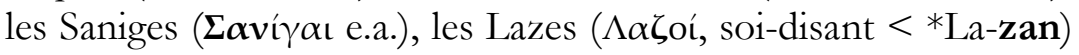

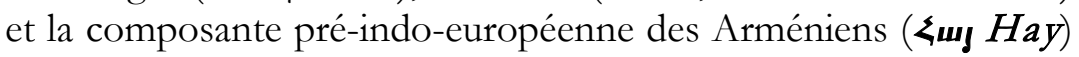
- dont la reconnaissance a par ailleurs été un élément capital dans le développement de la pensée de Marr. ${ }^{61}$ Autant de peuplades du Pont et du Caucase occidental, dont le rapprochement à cette échelle est rationnellement inconcevable. Comme le conclut lucidement Igor Diakonoff, " pour toutes ces raisons, s'efforcer d'établir l'ethnicité préhistorique d'un peuple en cherchant dans les temps anciens divers ethnonymes à la consonance similaire est une méthode peu fiable et qui conduit fréquemment à de considérables erreurs $\gg{ }^{62}$

\section{Répercussions modernes : le cas du philologue Elguža Xintibize}

Plus proche de nous encore que Toumanoff, le philologue géorgien Elguža Xintibize (*1937), dans un article paru en 2002, s'intéresse à l'étymologie des ethnonymes désignant les Géorgiens, au sujet desquels il propose une théorie nouvelle. Nous verrons qu'il y produit une série d'étymologies plus ou moins probables dans la lignée de Marr, mais parées d'atours nettement plus scientifiques. $^{63}$

Philologue bien connu en Géorgie, Xintibize s'est distingué par ses travaux sur la littérature géorgienne médiévale, étudiant en particulier l'œuvre de Šota Rustaveli, les textes philosophiques et les contacts littéraires entre la Géorgie et l'Occident. Ses recherches dans ces domaines sont d'une tenue scientifique tout à fait honorable, même si certaines des thèses qu'il défend ne font pas l'unanimité : l'identification du pseudo-Denys l'Aréopagite

${ }^{61}$ Sur la plupart de ces ethnonymes, voir notre article «Les Géorgiens dans les textes byzantins jusqu'à l'an mille. Approche lexicale ", à paraitre dans Byzantion 84 (2014)) ; pour la théorie du caractère mixte de la langue arménienne, voir en premier lieu THOMAS, Linguistic Theories, op. cit., p. 18-34.

${ }^{62} \mathrm{D}^{\prime} \mathrm{JAKONOV}$, Предыстория, ор. cit., p. 196.

${ }^{63}$ E. KHINTIBIDZE, «A New Theory on the Etymology of the Designations of the Georgians ", in W. BUBLITZ, M. VON RONCADOR et H. VATER (ed.), Philologie, Typologie und Sprachstruktur. Festschrift für Winfried Boeder zum 65.

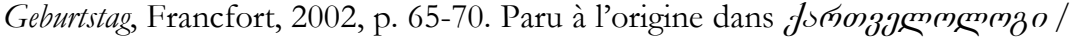
The Kartvelologist 9 (2001) et consultable en ligne à l'adresse http://www. kartvfund.org.ge/index.php?act $=$ page\&id $=33.58 \&$ lang $=$ en. . Cet article donne un condensé des idées défendues dans g. bobonobodj, fonomzgenons bsbjg-

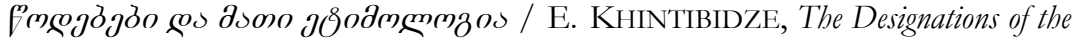
Georgians and Their Etymology, Tbilissi, 1998. Une analyse critique détaillée de cet ouvrage serait extrêmement profitable, mais un tel examen dépasserait largement le cadre que nous nous sommes ici fixé ; c'est pourquoi notre développement suivra celui, plus concis, de l'article de Xintibize - du reste plus aisément accessible au lecteur intéressé que sa monographie. 
avec le moine géorgien Pierre l'Ibère ${ }^{64}$ ou la spéculation d'une extraction géorgienne pour Basile de Césarée ${ }^{65}$ en sont des exemples.

Après une introduction destinée à familiariser le lecteur avec les exonymes (modernes) des Géorgiens et les postulats linguistiques à la base de son étude, Xintibize se lance dans l'identification mutuelle des différents démonymes. Il prétend en effet établir que toutes les dénominations connues pour les Géorgiens, dans toutes les langues, sont étymologiquement apparentées par leurs trois racines ultimes, qu'il cite sous les formes ber/ver, nel (vel) et kar et qu'il considère comme des "contreparties", ou des « formes linguistiques parallèles ». ${ }^{66}$

Son développement part de la racine ber identifiée dans 'I $\beta \eta{ }^{\prime} \alpha$, et la première chose qu'il y fasse est d'ailleurs d'invoquer l'autorité de Marr pour appuyer le découpage du mot en $i$ (formant) + ber (racine). Marr n'est plus cité dans la suite de l'article (sinon dans la bibliographie), mais son influence est patente dans des phrases comme "Among ancient peoples the wolf, as a totemic animal, gave rise to ethnonyms ", glissée de façon apparemment anodine, sans exemple ni référence. ${ }^{67}$ Etant donné que Xintibize se base sur les travaux de Tamaz Gamq'relize et Vjačeslav V. Ivanov promoteurs de la controversée théorie glottalique - pour ce qui dans sa théorie touche à la linguistique indo-européenne, l'on pourrait croire que cette affirmation dérive de ces derniers. Effectivement, l'ouvrage qu'ils ont coécrit propose une reconstitution du statut rituel du loup dans la société proto-indo-européenne et discute les rapports étymologiques de cette racine avec certains ethnonymes ainsi que ses rapports typologiques avec la culture kartvélienne. ${ }^{68}$ Toutefois, leurs vues sont plus mesurées que celles de Xintibize, notamment en ce qu'ils considèrent "probable» l'origine totémique des ethnonymes qu'ils mentionnent, tandis que Xintibize présente cela comme un fait, une donnée acquise. ${ }^{69}$

${ }^{64}$ E. KHINTIBIDZE, Georgian-Byzantine Literary Contacts, Amsterdam, 1996, p. $145-153$.

65 Ibid., p. 167-192.

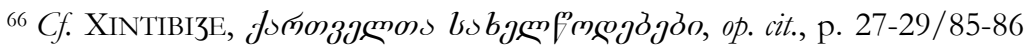
et 46-47/103-104.

${ }^{67}$ Les renvois aux travaux de Marr sont nettement plus fréquents dans XINTIBI3E, fృ๓о noms de personnes, Marr y est même l'auteur le plus souvent cité.

68 Th. V. GAMkRELIDZE - V. V. IVANOV, Indo-European and the IndoEuropeans, trad. du russe par J. Nichols (Trends in Linguistics, Studies and Monographs 80), Berlin / New York, 1995, vol. 1, p. 413-417.

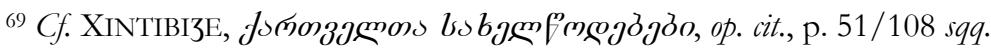


En 1905, Marr s'estimait en mesure de relier étymologiquement, au moyen d'une racine ber $<b r$, les termes 'I $\beta \eta q^{\alpha} \alpha$, dgrndj 60

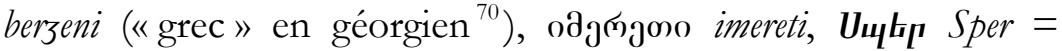
lıзgตo sp'eri (ville et district de T'ao; act. İspir, dans le district d'Erzurum en Turquie), зуy̆о guria (la Gourie, au nord de

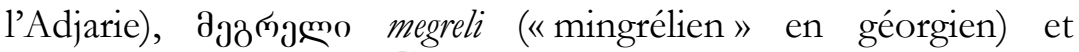
quelques autres encore. ${ }^{71}$ Quoique Marr ait considéré, à mesure de l'avancement de ses théories, ce type de relations entre lexèmes comme toujours plus complexe et moins génétique, cette allégation, même dépouillée de toute implication génétique, demeure véritablement nocive, car elle induit facilement des équivalences de signifié, qui sont au mieux impossibles à prouver, au pire contredites par le donné historique, littéraire ou archéologique.

Xintibize ne prend pas autant de termes en compte : exeunt notamment dgตndgfo berzeni et odgngono imereti, dont l'étymologie par une base ber identique à celle d'Ißßøí $\alpha$ est aujourd'hui proprement indéfendable. ${ }^{72}$ Par ailleurs, plutôt que de recourir à une explication froidement monogénétique, comme le faisait Marr au début de sa carrière, Xintibize reconnait un cycle d'ethnonymes interdépendants, articulé autour de trois racines unies par leur origine totémique, ainsi que par des possibilités d'alternances - d'une façon très proche de ce que proposait Marr aux stades ultérieurs de sa carrière. $^{73}$

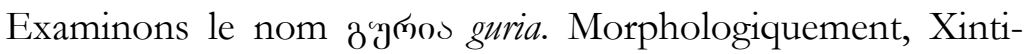

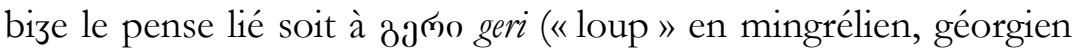
доgmo mgeli), soit au «radical indo-européen gurg». In fine, cela revient au même. En effet, Xintibize tire cet élément "gurg» du persan moderne (Gorji " géorgien », Gorjestān " Géorgie », gorg «loup »), la racine *wel-, qui aurait abouti aux noms perses des Géorgiens (not. via le moyen perse vir-č-, $\operatorname{var}(\mathrm{u})-\check{c}^{-}{ }^{74}$ ), constituant

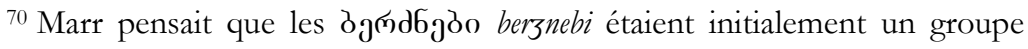
installé entre les Géorgiens et les Grecs, auxquels ce nom aurait été transféré par la suite.

${ }^{71}$ Détails dans ThOMAs, Linguistic Theories, op. cit., p. 12-13. Ces étymologies sont exposées pour la première fois dans MARR, Kpemenie, op. cit., p. 165 sqq.

${ }^{72}$ Ces ethnonymes sont en effet ignorés dans KHINTIBIDZE, New Theory, op.

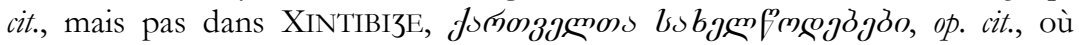
odğgon est bel et bien lié à «i-ber", «however, not on the questionable ground of sound gradation set forth in Japhetology» (p. 61/117), et de même pour ogndj6o (p. 47/104 sqq. et 64/120). Dans l'ensemble, on constate que l'auteur se montre plus prudent dans son article que dans sa monographie.

${ }^{73}$ De plus, la théorie de Xintibize entend relier les noms des Géorgiens à divers ethnonymes et toponymes célèbres d'Europe ( $c f$. e.a. XINTIBI3E, form-

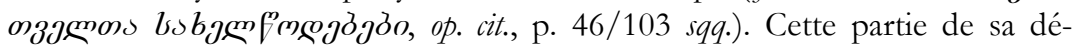
marche, sur laquelle nous ne nous étendrons pas, présente certaines affinités avec le travail de Marr dans sa phase «méditerranéenne ».

${ }^{74}$ Voir DeETERS, Name der Iberer, op. cit., p. 87. 
selon Xintibize, qui s'inspire de Gamq'relize et Ivanov, la racine « initiale indo-européenne désignant le loup ». ${ }^{75} \mathrm{D}$ 'un autre côté,

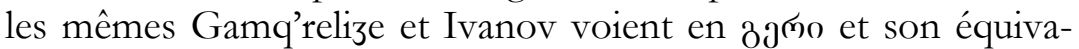

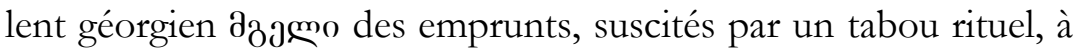
l'arménien quI gayl, de même sens, qui remonte lui aussi, selon eux, à cette racine ${ }^{*}$ wel-. ${ }^{76}$ Quoi qu'il en soit, l'étymologie de

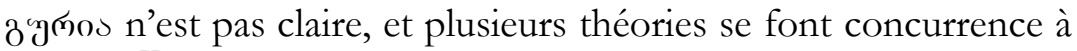
ce sujet. ${ }^{77}$ D'ailleurs, si le nom 3 ğ tème, c'est en premier lieu car il «semble être apparenté au nom totémique du loup »; les considérations étymologiques sont secondaires à ce rapprochement sémantique. Cela dit, Xintibize évoque ensuite une "alternance des complexes $b r$ et $g r$ », ce qui invite à rattacher gur- de présenté auparavant comme « une contrepartie de la racine $u e l »{ }^{78}$

En fait, Xintibize est exclusivement préoccupé de détecter des similitudes entre les diverses dénominations des Géorgiens. Les insuffisances de la méthode étymologique qui, même poussée jusqu'aux limites de ce qui est raisonnablement concevable, est incapable de ramener tout à l'unité, se voient palliées par diverses "équivalences", soit formelles ${ }^{79}$ soit conceptuelles ${ }^{80}$, qui permettent d'atteindre l'objectif tant convoité d'une unité fondamentale de ces dénominations. L'impression globale qui ressort des deux travaux de Xintibize est celle d'un appel ad hoc, sans discer-

75 Cf. GAMkrelidze - IVANov, Indo-European, op. cit., p. 413 n. 1. La reconstruction traditionnelle connait PIE *ứl $k^{\mathrm{u}}$-os, d'où e.a. gr. $\lambda u ́ \kappa o s, ~ l a t . ~ l u p u s$, vsl. в ъьь, skr. vớkas; les auteurs postulent, eux, une dérivation à partir de *wel- «lacérer », avec deux suffixes différents selon les dialectes du PIE. Contrairement à ce que laisse entendre la formulation de Xintibize, cette racine

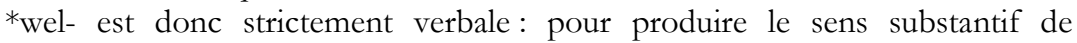
« loup », un suffixe est nécessaire.

76 GAMKRELIDZE - IVANOV, Indo-European, op. cit., p. 416 et 413. Une éty-

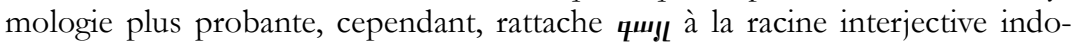
européenne *uai-, d'où e.a. lat. uae, gall. gwae, arm. «w vay : le nom du loup, litt. « le hurleur», est alors obtenu au moyen d'un suffixe *-lo-, à la fois en arménien et en celtique (gaulois, moyen-irlandais) (ainsi Hr. K. MARTIROSYAN, Etymological Dictionary of the Armenian Inherited Lexicon (Leiden Indo-European Etymological Dictionary Series 8), Leyde / Boston, 2010, p. 197-198 et J. POKORNY, Indogermanisches etymologisches Wörterbuch, Berne / Munich, 1959, vol. 1, p. 1110-1111).

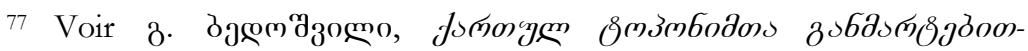

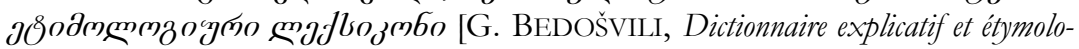
gique des toponymes géorgiens], t. 1, Tbilissi, 2002, p. 119-121.

${ }^{78}$ Davantage de détails sont présentés dans XiNTIBI3E, funonzJemons bs-

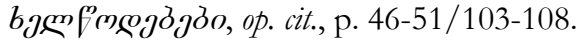

${ }^{79}$ P. ex. la correspondance de construction perçue entre $4 . / p$ - $p$ Vir-k' (nom arménien des Géorgiens et, par métonymie, de la Géorgie) et *j১๗ூ-go *kar-et, censément ancêtre de la racine fonnon-kart-.

${ }^{80}$ Comme nous l'avons vu pour les correspondances établies via le totem du loup. 
nement, aux outils les plus divers, dans le but d'étayer le mieux possible une théorie déjà acceptée au préalable. Dans ses détails, la théorie qu'il présente s'apparente de très près à ce qui avait été proposé par Marr et relayé par Toumanoff. ${ }^{81}$

\section{Conclusion}

Marr était un universaliste. Durant la majeure partie de sa vie, il se sera consacré, de différentes façons, à "décloisonner» sa Géorgie natale en lui découvrant une place centrale dans l'entrelacs linguistique mondial. La japhétidologie qu'il avait édifiée n'est plus; en revanche, il existe aujourd'hui d'autres disciplines bien établies, dans lesquelles s'insérerait partiellement le travail de Marr, à savoir la roustvélologie, la kartvélologie et la caucasologie. ${ }^{82}$ Or la naissance de ces disciplines doit justement aux «déviances » internationalistes de Marr, contre lesquelles certains de ses étudiants géorgiens ont réagi en proposant ces projets d'envergure plus restreinte. ${ }^{83}$ Par retour de balancier, ceux-ci n'ont depuis le départ pas été exempts de relents nationalistes, à divers degrés. Ainsi, l'intérêt des premiers caucasologues géorgiens (notamment le philologue Ivane క̌avaxišvili) dans le développement de cette nouvelle science allait à l'étude du contexte dans lequel s'est développée la culture géorgienne. Dans cette optique, l'élément kartvélien est central, les autres cultures du Caucase (arménienne, abkhaze, ossète, svane, turque, avare etc.) étant placées au service de sa compréhension. ${ }^{84}$

Cyrille Toumanoff a centré ses recherches sur ce qu'il a appelé la "Caucasie chrétienne », en tant qu'ensemble géographique et culturel cohérent profondément impliqué dans l'Orient médiéval et, plus largement, dans l'histoire mondiale. Cultures et sociétés arménienne et géorgienne sont donc indissociables et traitées sur un même pied - l'Albanie, troisième élément de cette Caucasie chrétienne, occupe une place mineure en raison du peu de traces qu'elle a laissées -, au point que l'on a pu qualifier le prince Toumanoff de «patriote caucasien » à sa propre façon. ${ }^{85}$

${ }^{81}$ MARr, Kpemenie, op. cit., p. 166-170 ; TOUMAnOFF, Social Background, op. cit., n. 58 p. 61 . Il est intéressant de constater que ce dernier exprime malgré tout quelques doutes, surtout pour ce qui concerne les implications linguistiques du culte du loup.

${ }^{82}$ CHERCHI - MANNING, Disciplines and Nations, op. cit., p. 6 et 54-55.

${ }^{83} \mathrm{Ibid}$., passim sur la kartvélologie et la caucasologie. Pour la roustvélologie, voir TUITE, Ibero-Caucasian Hypothesis, op. cit., p. 58-59.

${ }^{84}$ CHERCHI - MANNING, Disciplines and Nations, op. cit., p. 34-37, $42-44$ e.a.

${ }^{85}$ Hewsen, In Memoriam, op. cit., p. 7. Il est à remarquer que Slezkine, Soviet Ethnogenetics, op. cit., p. 836, qualifie de même le jeune Marr de "patriote pancaucasien ». 
La bibliographie d'Elguža Xintibize ${ }^{86}$ témoigne, elle, d'un intérêt pour la communauté culturelle de la Géorgie ancienne non avec le Caucase, mais avec l'Occident. Les interactions de ces deux régions sont étudiées, avec, comme nous avons pu le constater, une attention toute particulière portée, lorsque cela est possible, aux éléments géorgiens qui ont pénétré l'Occident, davantage encore qu'à l'influence de l'Occident en Géorgie.

Ce leitmotiv qui anime à la fois Marr, Toumanoff et Xintibize pourrait être qualifié de "pancaucasien » : chacun d'eux a voulu, à sa manière et dans son domaine, déployer la Géorgie au-delà de ses frontières. Que Toumanoff et Xintibize - linguistes ni l'un ni l'autre - aillent, à cette fin, puiser aux théories linguistiques de Marr, n'est guère surprenant. La célébrité de Marr, son extraction géorgienne, son idéologie universaliste et l'apparence scientifique de sa méthode constituent autant de raisons de faire appel, sans mauvaise conscience, à ses étymologies, qui prétendent en dire beaucoup plus sur l'antiquité géorgienne que nous n'en savons réellement.

A l'opposé de ce pancaucasisme abreuvé aux étymologies de Marr, il conviendrait d'évoquer un autre écueil, d'autant plus nocif qu'il connait davantage de répercussions en dehors du monde scientifique: nous voulons parler de l'interprétation nationaliste ou ethniciste du donné linguistique, ainsi que des textes littéraires et historiques. Certains ont en effet revendiqué, sans nuances, la " géorgianité » de tel peuple à telle époque par le biais d'une «superposition simpliste du territoire, de la langue, de l'ethnos et de la nation ${ }^{87}$. La disparition de l'Union Soviétique a catalysé de telles approches et les polémiques qui en découlent inévitablement; c'est précisément en 1991 que nous avons le meilleur exemple d'une telle controverse. ${ }^{88}$ Ici encore, ethnonymes et toponymes sont soumis à des étymologies qui témoignent des opinions politiques et idéologiques de leurs défenseurs, plutôt que de l'état réel de nos connaissances sur l'histoire ethnique, linguistique et culturelle de ces régions.

\footnotetext{
86 Une liste de titres majeurs est accessible en ligne, à l'adresse http://www. science.org.ge/members/pl/khintibidze.htm.

87 TUITE, Ibero-Caucasian Hypothesis, op. cit., p. 53.

88 B.G. HEWITT, « The valid and non-valid application of philology to history ", in Revue des Études Géorgiennes et Caucasiennes, 6-7 (1990-1991), p. 247-263. Pour un aperçu général de la question, voir TUITE, Ibero-Cancasian Hypothesis, op. cit., p. 53-68.
} 